\title{
Flat-band localization in Creutz superradiance lattices
}

\author{
Yanyan He, ${ }^{1, *}$ Ruosong Mao, ${ }^{1, *}$ Han Cai, ${ }^{1, \dagger}$ Jun-Xiang Zhang,,${ }^{1, \dagger \dagger}$ \\ Yongqiang Li, ${ }^{2}$ Luqi Yuan, ${ }^{3}$ Shi-Yao Zhu, ${ }^{1}$ and Da-Wei Wang ${ }^{1,4}$ \\ ${ }^{1}$ Interdisciplinary Center for Quantum Information, \\ State Key Laboratory of Modern Optical Instrumentation, \\ and Zhejiang Province Key Laboratory of Quantum Technology and Device, \\ Department of Physics, Zhejiang University, Hangzhou 31002\%, Zhejiang Province, China; \\ ${ }^{2}$ Department of Physics, National University of Defense Technology, Changsha 410073, Hunan Province, China; \\ ${ }^{3}$ State Key Laboratory of Advanced Optical Communication Systems and Networks, \\ School of Physics and Astronomy, Shanghai Jiao Tong University, Shanghai 200240, China; \\ ${ }^{4}$ CAS Center for Excellence in Topological Quantum Computation, \\ University of Chinese Academy of Sciences, Beijing 100190, China
}

(Dated: October 15, 2020)

\begin{abstract}
Flat bands play an important role in diffraction-free photonics and attract fundamental interest in many-body physics. Here we report the engineering of flat-band localization of collective excited states of atoms in Creutz superradiance lattices with tunable synthetic gauge fields. Magnitudes and phases of the lattice hopping coefficients can be independently tuned to control the state components of the flat band and the Aharonov-Bohm phases. We can selectively excite the flat band and control the flat-band localization with the synthetic gauge field. Our study provides a room-temperature platform for flat bands of atoms and holds promising applications in exploring correlated topological materials.
\end{abstract}

Flat bands are characterized by the zero bandwidth over the whole Brillouin zone. Owing to the destructive interference between the hopping pathways [1, 2], the group velocity of excitations vanishes, and hence the diffusion in flat bands is inhibited. The resulting compact localized eigenstates (CLSs) [3, 4] have been experimentally realized in photonics $5-13$ and polariton-exciton condensates [14, 15]. Immune to environmental noises, localized states in flat bands are promising candidates for realizing quantum networks [16] and diffraction-free photonics [17-19]. Flat bands are also of fundamental interest in many-body physics because of their high degeneracy. The density of states is divergent such that even weak interactions lead to strong correlations and exotic topological phases [20 23].

Many-body interactions can be engineered to realize correlated topological phases in atoms [24, 25]. However, in previous realizations of the flat bands in optical lattices, the underlying lattices [26, 27] are gapless and topologically trivial. A feasible model that integrates both band flatness and topology is the two-leg ladder in a uniform magnetic field with cross-linked couplings, i.e., the Creutz lattice 28, 29] (see Fig. 1(a)). Despite theoretical proposals in photonic waveguides [9] and ultracold atoms [21], flat bands in the Creutz lattice have never been experimentally realized $30-32$.

Here we report the synthesis of a Creutz ladder with tunable tight-binding parameters in the form of a momentum-space lattice, i.e., the superradiance lattice [33, 34, in room-temperature cesium atoms. The bipartite ladder consists of timed Dicke states with different momenta [35]. We find that the corresponding energy band structure exhibits a flat band and a dispersive band, which are distinguished by localized and delocalized ex- citations, respectively. In the experiment, we excite one site in the ladder with a weak probe field and measure the optical response of the adjacent site. The hopping strengths and the Aharonov-Bohm (AB) phases in the lattice are carefully tuned, which enables us to excite a particular band and control the flat-band localization. We observe that the optical response is significantly suppressed when the flat band is selectively excited. By controlling the $\mathrm{AB}$ phases, we reveal the relation between the flat-band localization and gauge fields [36. Our work demonstrates a versatile platform for flat bands of atoms with multiple tunable parameters, which holds promising applications in exploring correlated topological phases.

We first introduce the experimental scheme implemented in the hyperfine levels of the ${ }^{133} \mathrm{Cs}$ D1 line in a bichromatic standing-wave-coupled configuration, as shown in Fig. 1(a). Two standing waves couple two excited states $|a\rangle \equiv\left|6^{2} P_{1 / 2}, F=3\right\rangle$ and $|b\rangle \equiv$ $\left|6^{2} P_{1 / 2}, F=4\right\rangle$ to the same metastable state $|c\rangle \equiv$ $\left|6^{2} S_{1 / 2}, F=3\right\rangle$. The frequency of the $j$ th standing-wave coupling field $\nu_{j}$ fulfills the two-photon resonance condition $\Delta_{c}=\nu_{1}-\omega_{a c}=\nu_{2}-\omega_{b c}$, where $\omega_{i j}$ being the atomic transition frequency between $|i\rangle$ and $|j\rangle$. The envelopes of the Rabi frequency amplitude of the two standing waves are $2 \Omega_{1} \cos \left(k_{c} x-\phi / 4\right)$ and $2 \Omega_{2} \cos \left(k_{c} x+\phi / 4\right)$, where $k_{c}$ is the $x$ component of the wave vectors and $\phi / 2$ is the phase difference between the envelopes. The wave-vector difference between the two standing waves is negligible in the length of the atomic vapor cell. We use a weak travelling field with the wave vector $k_{p}$ to probe the standing-wavecoupled atomic vapor and measure the backward reflection. The frequency of the probe field $\nu_{p}$ is scanned to couple the ground state $|g\rangle \equiv\left|6^{2} S_{1 / 2}, F=4\right\rangle$ to either the state $|a\rangle$ or $|b\rangle$. Featured signals can be observed 

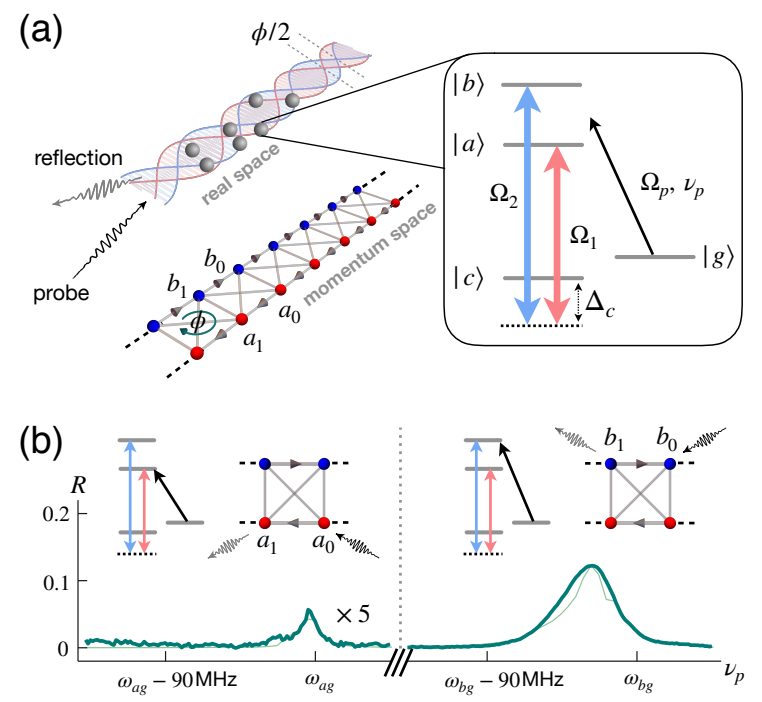

Figure 1. (a) Schematic configuration of the experiment. The amplitude envelopes of the two standing waves have a $\phi / 2$ phase difference. In the Creutz ladder, the arrows indicate the phase $\phi / 2$ attached to the transitions. Inset is the configuration of atomic levels and laser fields. (b) Typical reflection spectrum. The insets shows the configurations of the fields and the lattice responses when $\nu_{p}$ is near resonant with either atomic transitions. The $\mathrm{AB}$ flux $\phi=\pi$. The power of the probe field is $24 \mu \mathrm{W}$. The powers of each plane wave component of the two standing waves are $P_{1}=29 \mathrm{~mW}$ and $P_{2}=215 \mathrm{~mW}$ with effective Rabi frequencies $\Omega_{1}=15 \mathrm{MHz}$ and $\Omega_{2}=68 \mathrm{MHz}$. The thick dark (thin light) lines are the experimental data (numerical simulation).

when the probe field is near resonant with each atomic transition. A typical spectrum is shown in Fig. 1(b).

In order to show that our experiment constructs a Creutz ladder and reveal the connection between the reflection signal and the excitation transport in the ladder, we write the Hamiltonian $H=H_{s}+H_{p}$ in momentum space [37, with $H_{s}$ and $H_{p}$ being the parts of the Hamiltonian corresponding to the couplings of standing waves and the probe field, respectively. Here, we set $\hbar=1$ and $H_{s}$ reads

$$
\begin{aligned}
H_{s} & =\sum_{n}\left[2 t_{1} a_{n}^{\dagger} a_{n}+2 t_{2} b_{n}^{\dagger} b_{n}\right. \\
& +\left(2 t_{3} \cos \frac{\phi}{2} a_{n}^{\dagger} b_{n}+t_{3} a_{n}^{\dagger} b_{n+1}+t_{3} b_{n}^{\dagger} a_{n+1}\right. \\
& \left.\left.+t_{1} e^{-i \phi / 2} a_{n}^{\dagger} a_{n+1}+t_{2} e^{i \phi / 2} b_{n}^{\dagger} b_{n+1}+\text { h.c. }\right)\right],
\end{aligned}
$$

which gives a tight-binding superradiance lattice composed of the collective atomic excitation operators $d_{j}^{\dagger}=$ $\sqrt{1 / N} \sum_{m}\left|d_{m}\right\rangle\left\langle g_{m}\right| \exp \left[i\left(k_{p}-2 j k_{c}\right) x_{m}\right](d=a, b)$ [33], where $m$ labels the $m$ th atom at the position $x_{m}, j$ is an integer, and $N$ is the total number of atoms. $t_{1}=-\Omega_{1}^{2} / \Delta_{c}$ and $t_{2}=-\Omega_{2}^{2} / \Delta_{c}$ are the hopping amplitudes along $a$-leg and $b$-leg, respectively. Here, we can adiabatically eliminate the state $|c\rangle$, since $\Delta_{c}$ is much larger than all relevant Rabi frequencies $\left(\Delta_{c} \gg \Omega_{j}\right)$. The two hoppings acquire a phase $\phi / 2$ in opposite directions. The loop transition along a plaquette accumulates an $\mathrm{AB}$ phase $\phi$, such that the lattice is effectively in a uniform magnetic field. $t_{3}=-\Omega_{1} \Omega_{2} / \Delta_{c}$ and $2 t_{3} \cos \phi / 2$ are the hopping strengths along the diagonals and the rungs of each plaquette in the ladder. The on-site energies of the $a$-leg and $b$-leg sites are $2 t_{1}$ and $2 t_{2}$, respectively.

The probe field coupling Hamiltonian is $H_{p}=$ $\sqrt{N} \Omega_{p} e^{-i \Delta_{p}^{\prime} t} a_{0}^{\dagger}+\sqrt{N} \Omega_{p}^{\prime} e^{-i \Delta_{p} t} b_{0}^{\dagger}+h . c$. , where $\Omega_{p}\left(\Omega_{p}^{\prime}\right)$ and $\Delta_{p}=\nu_{p}-\omega_{b g}\left(\Delta_{p}^{\prime}=\nu_{p}-\omega_{a g}\right)$ are the Rabi frequency and the frequency detuning of the coupling between the probe field and the atomic transition between $|a\rangle(|b\rangle)$ and $|g\rangle$. Hence, $H_{p}$ shows that the excitation is prepared by the probe field to the site $a_{0}$ or $b_{0}$ in the ladder. When we probe the site $a_{0}\left(b_{0}\right)$, the phase-matching condition $k_{p}-2 k_{c} \approx-k_{p}$ is only satisfied for the excitation on the site $a_{1}\left(b_{1}\right)$, which results in a superradiant backward emission collected by a photodetector. The spectrum in the left (right) of Fig. 1(b) characterizes the excitation transport from $a_{0}$ to $a_{1}\left(b_{0}\right.$ to $\left.b_{1}\right)$ in the ladder of Eq. (1). In the experiment, the probe field is weak $\left(\Omega_{p} \ll t_{i}\right)$ such that only a small fraction of the atoms are excited. In this condition, $a_{j}, b_{j}$ are approximately bosonic annihilation operators [33].

The Creutz ladder in Eq. (1) constructed in momentum space is tunable in the experiment. We diagonalize $H_{s}$ in real space [37] and the band structures are shown in Fig. 2. We define $\eta \equiv t_{2} / t_{1}$ as the relative hopping strength along the two legs. One can see that all three band structures with different $\eta$ are composed of a flat band and a dispersive band. In general, the dynamics of the excitation is governed by both bands and cannot be distinguished. Probing only one band by controlling the excitation energy [41,43] is inapplicable since the band gap closes when $\phi$ approaches zero [37].

Nevertheless, the tunability of the hopping strengths enables us to determine which band to excite by controlling the state component of the bands. In the experiment, we tune $\eta$, which is proportional to $P_{2} / P_{1}$, where $P_{1}$ and $P_{2}$ are the powers of the two standing waves. An interesting correlation can be noticed between the parameter $\eta$ and the band components, where the color represents the polarization $\left\langle\sigma_{z}\right\rangle \equiv\langle\mid a\rangle\langle a|-| b\rangle\langle b \mid\rangle$ of the eigenstates. In particular, $\left\langle\sigma_{z}\right\rangle=+1$ or -1 means the band fully locates on the $a$ - or $b$-leg. In Fig. 2(a), one can see that $\left\langle\sigma_{z}\right\rangle \approx-1$ for almost the whole dispersive band, meaning that the dispersive band supports a large excitation component on the $b$-leg for $\eta \gg 1$ (we take $\eta=20.7$ according to the experimental parameters). Therefore, the $b_{0} \rightarrow b_{1}$ transport dynamics is governed by the dispersive band. On the other hand, for $\eta \ll 1(\eta=1 / 20.7$ as shown in $2(\mathrm{c})),\left\langle\sigma_{z}\right\rangle \approx-1$ for almost the entire flat band, so the $b_{0} \rightarrow b_{1}$ transport dynamics is governed by the flat band.

This band selection is manifested in the bandwidth, 


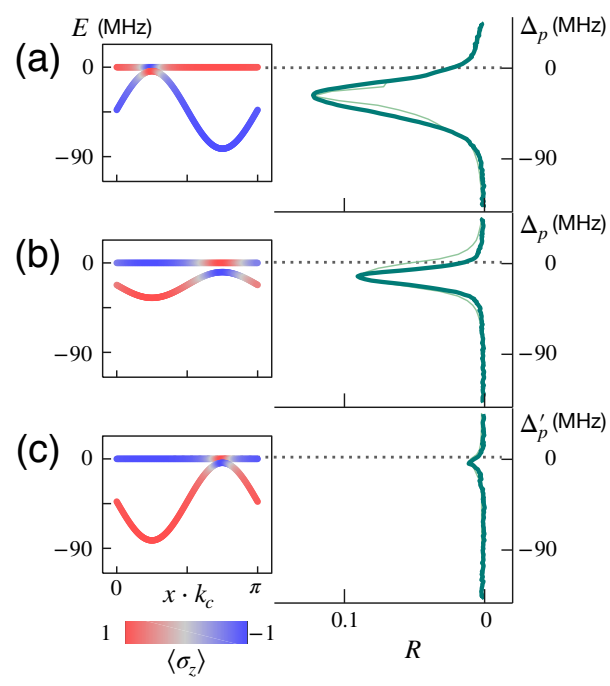

Figure 2. Band structures (left) and the corresponding reflection spectra (right) with (a) $\eta=20.7$, (b) $\eta=4.0$, and (c) $\eta=1 / 20.7$. The reflection spectrum is mainly contributed by the dispersive (flat) band for $\eta \gg 1(\eta \ll 1)$. The data in (c) is obtained by measuring the $a$-leg response owing to the symmetry of the Hamiltonian (see the text). Dotted gray lines mark the energy of the flat band.

the central frequency, and the magnitude of the reflection spectrum in Fig. 2. In the experiment, we change $\eta$ and keep $t_{3} \propto \sqrt{P_{1} P_{2}}$ a constant. In Fig. 2(a) for $\eta \gg 1$, the reflection spectrum of the dispersive band has a larger bandwidth and a lower central frequency. As a comparison, in Fig. 2(c) for $\eta \ll 1$, the reflection spectrum due to the flat band has a much narrower bandwidth and the peak locates near the predicted frequency of the flat band. The localization in the flat band is demonstrated by the decrease of the reflection peak when we decrease $\eta$, during which the reflection is more contributed by the flat band.

As a side note, in obtaining Fig. 2(c), we use the symmetry that lattice Hamiltonian $H_{s}$ is invariant when we exchange the sublattices $a$ and $b$, inverse $\eta$, and flip the flux $\phi$ [37]. In the experiment, the $b_{0} \rightarrow b_{1}$ transport dynamics with $\phi$ and $\eta>1$ is characterized by the reflection spectrum near resonant with level $|b\rangle$ (labelled with $\left.R_{\eta}(\phi)\right)$, while the one with $-\phi$ and $(1 / \eta)<1$ is effectively obtained from the $|a\rangle$-side reflection spectrum (labelled with $R_{1 / \eta}(-\phi)$ ).

The reflection spectrum is mostly contributed by the slowly moving atoms that have Doppler shifts smaller than the lattice bandwidth [44. We take the average of reflectivity over the bands, i.e., $\bar{R}=\int R d \nu_{p} / \int d \nu_{p}$, to investigate the localization and its dependence on $\phi$. In Fig. 3(a), the flat-band localization is demonstrated by the suppression of $\bar{R}$ when $\eta$ decreases. Furthermore, we notice that the $\phi$-dependence of $\bar{R}$ changes with $\eta$ (see the $\phi$ calibration in [37]). The sinusoidal curve of averaged reflectivity $\bar{R}_{\eta}(\phi)$ is shifted from top to bottom
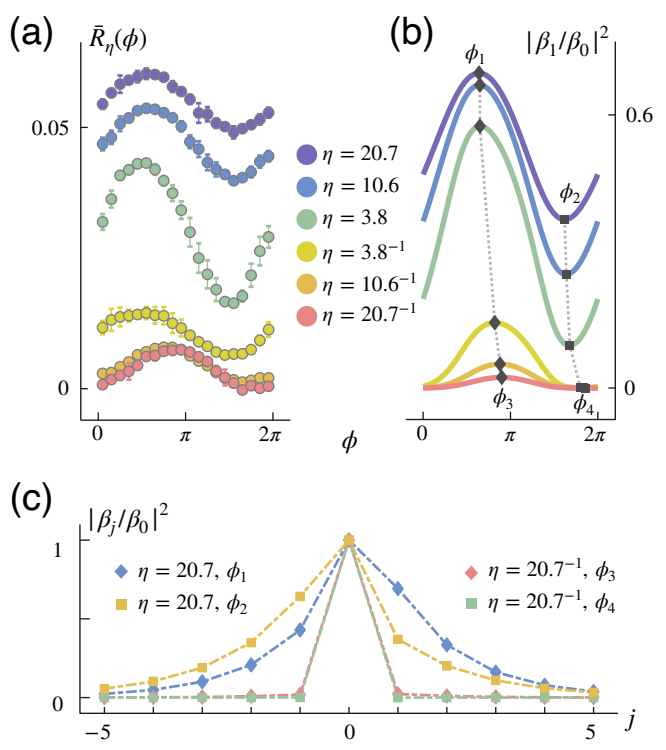

Figure 3. Response of the dispersive and flat bands with different gauge fields. (a) The averaged reflectivity $\bar{R}$ versus $\phi$ with different $\eta$. (b) The normalized probability on the site $b_{1}$ versus $\phi$. The diamonds (squares) indicate where the curves reach their maxima (minima). $\Delta_{p}\left(\Delta_{p}^{\prime}\right)=-2 \mathrm{MHz}$ and $\Delta_{c}=233.5 \mathrm{MHz}$. The gray dashed lines are plotted to guide the extrema of the curves. (c) The probability distribution of the steady state $\left|\psi_{s}\right\rangle$ on the $b$-leg with $\phi_{1}=0.64 \pi, \eta=20.7$ (blue diamonds), $\phi_{2}=1.62 \pi, \eta=20.7$ (yellow squares), $\phi_{3}=$ $0.9 \pi, \eta=1 / 20.7$ (red diamonds), and $\phi_{4}=1.86 \pi, \eta=1 / 20.7$ (green squares). The powers of each plane wave component of the two standing waves are $P_{1}=67 \mathrm{~mW}, P_{2}=90 \mathrm{~mW}$ for $\eta=3.8$ and $1 / 3.8 ; P_{1}=40 \mathrm{~mW}, P_{2}=153 \mathrm{~mW}$ for for $\eta=10.6$ and $1 / 10.6$; and $P_{1}=29 \mathrm{~mW}, P_{2}=215 \mathrm{~mW}$ for $\eta=20.7$ and $1 / 20.7$. Other experimental parameters are the same as in Fig. 1. The points are simply connected for clarity. Error bars are obtained from four independent data sets.

\section{in Fig. 3(a).}

The $\phi$-dependent shift shows the distinct responses to the gauge fields of the two bands. We consider two ideal cases to explain the physics. If the excitation is completely prepared in the dispersive band, the transport dynamics is determined by the flux-dependent unidirectional chiral edge current [44 51] of the dispersive band. The unidirectional chiral current breaks the symmetry between the transition from $b_{0}$ to $b_{1}$ and the one from $b_{0}$ to $b_{-1}$. When the magnetic flux $\phi \in(0, \pi)$, the chiral current enhances the probability in the site $b_{1}$, and hence the reflectivity increases (vice versa). On the other hand, flat-band response to the gauge field can be understood by the CLSs $\left|F_{j}\right\rangle$ [3]. When $\phi=2 n \pi$ ( $n$ is an integer $),\left|F_{j}\right\rangle \propto\left(\eta a_{j}^{\dagger}-b_{j}^{\dagger}\right)|G\rangle$ is localized within the $j$ th unit cell. Therefore, only $\left|F_{0}\right\rangle$ is excited when we probe the site $b_{0}$, leading to the maximum localization. Otherwise, $\left|F_{j}\right\rangle \propto\left(\eta a_{j+1}^{\dagger}-e^{i \phi / 2} b_{j+1}^{\dagger}+\eta e^{i \phi / 2} a_{j}^{\dagger}-b_{j}^{\dagger}\right)|G\rangle$ is localized within two unit cells. Probing site $b_{0}$ leads to a coherent superposition between $\left|F_{0}\right\rangle$ and $\left|F_{-1}\right\rangle$, and 
(a)

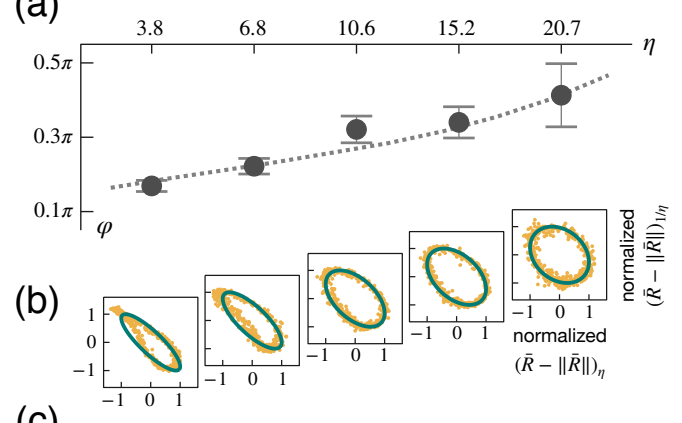

(c)

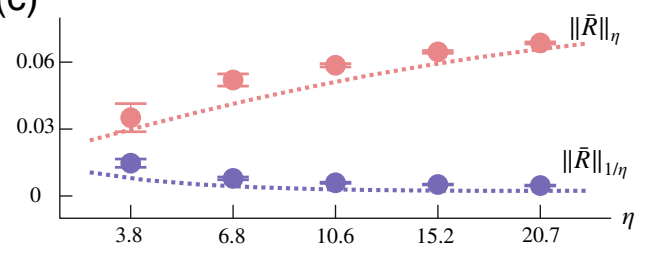

Figure 4. Gauge field dependence of the reflectivities from the dispersive band and flat band. (a) The phase difference $\varphi$ between the $\phi$-dependences of $\bar{R}_{\eta}$ and $\bar{R}_{1 / \eta}$. (b) The Lissajous curves composed of $\bar{R}_{\eta}(\phi)$ and $\bar{R}_{1 / \eta}(-\phi)$. The phase difference between $\bar{R}_{\eta}$ and $\bar{R}_{1 / \eta}$ increases and approaches $\pi / 2$ when $\eta$ increases. The yellow dots are experimental data and the green lines are fitted elliptic curves. The powers of each plane wave component of the two standing waves are $P_{1}=50 \mathrm{~mW}, P_{2}=124 \mathrm{~mW}$ for $\eta=6.8$ and $1 / 6.8$; $P_{1}=33 \mathrm{~mW}, P_{2}=183 \mathrm{~mW}$ for $\eta=15.2$ and $1 / 15.2$. Other experimental parameters are the same as in Fig. 3. Each plot contains 400 data points. (c) The mean averaged reflectivity $\|\bar{R}\|_{\eta}$ versus $\eta$. $\|\bar{R}\|_{\eta}$ increases with $\eta$ monotonically. The dotted lines are the simulated results.

hence results in a finite overlap with $b_{ \pm 1}$, which is similar to the "breathing motions" in Refs. [9, 10.

The two responses can be further investigated by the steady state of the collective excited states of atoms, where the wave function $\left|\psi_{s}\right\rangle \approx\left[1+\sum_{j}\left(\alpha_{j} a_{j}^{\dagger}+\beta_{j} b_{j}^{\dagger}\right)\right]|G\rangle$ $\left(\alpha_{j}, \beta_{j} \ll 1\right)$ in the weak excitation approximation. In the steady state, we obtain the probability amplitude $\beta_{j}=\sqrt{N} \Omega_{p}\left\langle G\left|b_{j}\left(\Delta_{p}+i \hat{\gamma}-H_{s}\right)^{-1} b_{0}^{\dagger}\right| G\right\rangle$ [52], where $\hat{\gamma}=\sum_{j}\left(\gamma_{a} a_{j}^{\dagger} a_{j}+\gamma_{b} b_{j}^{\dagger} b_{j}\right)$ and $\gamma_{a}\left(\gamma_{b}\right)$ is the decoherence rate between the excited state $|a\rangle(|b\rangle)$ and $|g\rangle$. Especially, we plot $\left|\beta_{1} / \beta_{0}\right|^{2}$, i.e., the normalized probability on the site $b_{1}$ in Fig. 3(b). Since the reflectivity is approximately proportional to $\left|\beta_{1} / \beta_{0}\right|^{2}[53$, both features of the $\bar{R}$ in Fig. 3.a) are demonstrated, including the magnitude suppression and the $\phi$-dependence. We also plot four typical probability amplitude distributions along the $b$-leg in Fig. 3(c) with $\eta \gg 1$ and $\eta \ll 1$. For $\eta=20.7$, the distributions of $\left|\beta_{j}\right|^{2}$ with $\phi_{1}=0.64 \pi$ and $\phi_{2}=1.62 \pi$ are almost symmetric to each other with respect to the 0th site, implying flux-dependent unidirectional chiral edge current of the dispersive band. However, the distributions with $\phi_{3}=0.9 \pi$ and $\phi_{4}=1.86 \pi$ are symmetrically localized at $b_{0}$ with different localization lengths. $\left|\beta_{1} / \beta_{0}\right|^{2}$ for $\eta=1 / 20.7$ is minimized at $\phi_{4}$, which means that the eigenstates are maximally localized when the synthetic gauge field almost vanishes. We notice that $\phi_{4}$ is slightly different from $2 n \pi$, which comes from the suppressed but finite contribution from the dispersive band.

In Fig. 4(b), the different $\phi$-dependences of the dispersive and flat bands are illustrated with Lissajous curves. The spatial phase difference between the envelopes of the two standing waves is slowly tuned to cover all values of $\phi$. We obtain the data sets $\left\{\bar{R}_{\eta}(\phi), \bar{R}_{1 / \eta}(-\phi)\right\}$ and fit them with ellipses to reconstruct the Lissajous curves. The shape of the ellipse elucidates the phase differences between the two underlying functions. For example, a Lissajous curve composed of two parametric equations with argument $u$, e.g., $x=\sin (u)$ and $y=\sin (-u+\varphi)$, is a line (circle) when $\varphi=0(\pi / 2)$. In Fig. 4 (a), $\varphi$ obtained by fitting the Lissajous curve approaches $\pi / 2$ when $\eta$ increases, indicating the different types of the excitations on the flat and dispersive bands. In Fig. 4(c), we notice that $\|\bar{R}\|_{\eta}$ increases with $\eta$ monotonically, where $\|$.$\| in-$ dicates the mean value of the averaged reflectivity $\bar{R}$ over all $\phi$. The numerical simulation agrees with the data.

In conclusion, we experimentally realize Creatz ladders with tunable gauge fields, where the flat band can be selectively excited and the interplay between the flatband localization and the $\mathrm{AB}$ phases was investigated. We study the flat-band localization in an open system, where the steady state balanced by pumping, driving, and dissipation exhibits the dynamics in the corresponding closed system [52]. We also need to emphasize that our scheme is substantially different from the incoherently pumped polariton-exciton condenstates [14, 15, 54, 56], where coherence is not accessible between multiple CLSs. It is interesting to notice that both bands of the Creutz ladder are topologically non-trivial [10, 31] provided that $\phi \neq 2 n \pi$ 37. It is a step towards the simulation of the strong correlated quantum phases, including the fractional Chern insulators [20, disorder-free many-body localization [57, and unusual ferromagnetism [58]. An interaction term between the sites in momentum space can be introduced by weakly coupling the excited atomic level to a Rydberg state [25] or by $s$-wave interactions [39, 40]. With a negative $\Delta_{c}$, the flat band has the lowest energy and can be used to study the many-body ground states of ultracold atoms [25, 37].

We acknowledge the support from the National Natural Science Foundation of China (Grants No. 11934011, No. 11874322, No. 91736209 and No. U1330203), the National Key Research and Development Program of China (Grants No. 2019YFA0308100 and No. 2018YFA0307200), and the Fundamental Research Funds for the Central Universities.

*These authors contributed equally to this work.

†hancai@zju.edu.cn

†junxiang_zhang@zju.edu.cn 
[1] B. Sutherland, Localization of electronic wave functions due to local topology, Phys. Rev. B 34, 5208 (1986).

[2] D. L. Bergman, C. Wu, and L. Balents, Band touching from real-space topology in frustrated hopping models, Phys. Rev. B 78, 125104 (2008).

[3] S. Flach, D. Leykam, J. D. Bodyfelt, P. Matthies, and A. S. Desyatnikov, Detangling flat bands into Fano lattices, Europhys. Lett. 105, 30001 (2014).

[4] D. Leykam, A. Andreanov, and S. Flach, Artificial flat band systems: from lattice models to experiments, Advances in Physics: X 3, 1473052 (2018).

[5] R. A. Vicencio, C. Cantillano, L. Morales-Inostroza, B. Real, C. Mejía-Cortés, S. Weimann, A. Szameit, and M. I. Molina, Observation of localized states in Lieb photonic lattices, Phys. Rev. Lett. 114, 245503 (2015).

[6] S. Mukherjee, A. Spracklen, D. Choudhury, N. Goldman, P. Öhberg, E. Andersson, and R. R. Thomson, Observation of a localized flat-band state in a photonic Lieb lattice, Phys. Rev. Lett. 114, 245504 (2015).

[7] S. Mukherjee and R. R. Thomson, Observation of localized flat-band modes in a quasi-one-dimensional photonic rhombic lattice, Opt. Lett. 40, 5443 (2015).

[8] S. Mukherjee and R. R. Thomson, Observation of robust flat-band localization in driven photonic rhombic lattices, Opt. Lett. 42, 2243 (2017).

[9] S. Mukherjee, M. D. Liberto, P. Öhberg, R. R.Thomson, and N. Goldman, Experimental Observation of Aharonov-Bohm Cages in Photonic Lattices, Phys. Rev. Lett. 121, 075502 (2018).

[10] M. Kremer, I. Petrides, E. Meyer, M. Heinrich, O. Zilberberg, and A. Szameit, A square-root topological insulator with non-quantized indices realized with photonic Aharonov-Bohm cages, Nat. Commum. 11, 1808 (2020).

[11] S. Xia, Y. Hu, D. Song, Y. Zong, L. Tang, and Z. Chen, Demonstration of flat-band image transmission in optically induced Lieb photonic lattices, Opt. Lett. 41, 1435 (2016).

[12] S. Xia, D. Li, X. Liu, L. Tang, Y. Hu, D. Song, J. Xu, D. Leykam, S. Flach, and Z. Chen, Unconventional flatband line states in photonic Lieb lattices, Phys. Rev. Lett. 121, 263902 (2018).

[13] J. Ma, J. W. Rhim, L. Tang, S. Xia, H. Wang, X. Zheng, S. Xia, D. Song, Y. Hu, Y. Li, B. J. Yang, D. Leykam, and Z. Chen, Direct observation of flatband loop states arising from nontrivial real-space topology, Phys. Rev. Lett. 124, 183901 (2020).

[14] F. Baboux, L. Ge, T. Jacqmin, M. Biondi, E. Galopin, A. Lemaître, L. Le Gratiet, I. Sagnes, S. Schmidt, H. E. Türeci, A. Amo, and J. Bloch, Bosonic condensation and disorder-induced localization in a flat band, Phys. Rev. Lett. 116, 066402 (2012).

[15] T. H. Harder, O. A. Egorov, J. Beierlein, P. Gagel, J. Michl, M. Emmerling, C. Schneider, U. Peschel, S. Höfling, and S. Klembt, Exciton-polaritons in flatland: Controlling flatband properties in a lieb lattice, arXiv: 2002.05405.

[16] M. Röntgen, C. Morfonios, I. Brouzos, F. Diakonos, and P. Schmelcher, Quantum network transfer and storage with compact localized states induced by local symmetries, Phys. Rev. Lett. 123, 080504 (2019).

[17] S. Rojas-Rojas, L. Morales-Inostroza, R. A. Vicencio, and A. Delgado, Quantum localized states in photonic flat-band lattices, Phys. Rev. A 96, 043803 (2017).

[18] R. A. Vicencio and C. Mejía-Cortés, Diffraction-free image transmission in kagome photonic lattices, Journal of Optics 16, 015706 (2013).

[19] D. Yu, L. Yuan, and X. Chen, Isolated photonic flatband with the effective magnetic flux in a synthetic space including the frequency dimension, arXiv: 2004.12542.

[20] E. J. Bergholtz and Z. Liu, Topological flat band models and fractional Chern insulators, Int. J. Mod. Phys. B 27, 1330017 (2013).

[21] J. Jünemann, A. Piga, S. J. Ran, M. Lewenstein, M. Rizzi, and A. Bermúdez, Exploring interacting topological insulators with ultracold atoms: The synthetic Creutz-Hubbard model, Phys. Rev. X 7, 031057 (2017).

[22] E. M. Spanton, A. A. Zibrov, H. Zhou, T. Taniguchi, K. Watanabe, M. P. Zaletel, and A. F. Young, Observation of fractional Chern insulators in a van der Waals heterostructure, Science 360, 62 (2018).

[23] G. Chen, A. L. Sharpe, E. J. Fox, Y. H. Zhang, S. Wang, L. Jiang, B. Lyu, H. Li, K. Watanabe, T. Taniguchi, Z. Shi, T. Senthil, D. Goldhaber-Gordon, Y. Zhang, and F. Wang, Tunable correlated Chern insulator and ferromagnetism in a moiré superlattice, Nature 579, 56 (2020).

[24] I. Bloch, J. Dalibard, and W. Zwerger, Many-body physics with ultracold gases, Rev. Mod. Phys. 80, 885 (2008).

[25] Y. Li, H. Cai, D. W. Wang, L. Li, J. Yuan, and W. Li, Many-body chiral edge currents and sliding phases of atomic spin waves in momentum-space lattice, Phys. Rev. Lett. 124, 140401 (2020).

[26] G. B. Jo, J. Guzman, C. K. Thomas, P. Hosur, A. Vishwanath, and D. M. Stamper-Kurn, Ultracold atoms in a tunable optical kagome lattice, Phys. Rev. Lett. 108, 045305 (2011).

[27] S. Taie, H. Ozawa, T. Ichinose, T. Nishio, S. Nakajima, and Y. Takahashi, Coherent driving and freezing of bosonic matter wave in an optical Lieb lattice, Sci. Adv. 1, e1500854 (2015).

[28] M. Creutz, End states, ladder compounds, and domainwall fermions, Phys. Rev. Lett. 83, 263617 (1999).

[29] D. Hügel and B. Paredes, Chiral ladders and the edges of quantum Hall insulators, Phys. Rev. A 89, 023619 (2014).

[30] J. H. Kang, J. H. Han, and Y. Shin, Realization of a cross-linked chiral ladder with neutral fermions in a $1 \mathrm{D}$ optical lattice by orbital-momentum coupling, Phys. Rev. Lett. 121, 150403 (2018).

[31] J. H. Kang, J. H. Han, and Y. Shin, Creutz ladder in a resonantly shaken $1 \mathrm{D}$ optical lattice, New J. Phys. 22, 013023 (2020).

[32] H. Alaeian, C. W. S. Chang, M. V. Moghaddam, C. M. Wilson, E. Solano, and E. Rico, Creating lattice gauge potentials in circuit QED: The bosonic Creutz ladder, Phys. Rev. A 99, 053834 (2019).

[33] D. W. Wang, R. B. Liu, S. Y. Zhu, and M. O. Scully, Superradiance lattice, Phys. Rev. Lett. 114, 043602 (2015).

[34] L. Chen, P. Wang, Z. Meng, L. Huang, H. Cai, D. W. Wang, S. Y. Zhu, and J. Zhang, Experimental observation of one-dimensional superradiance lattices in ultracold atoms, Phys. Rev. Lett. 120, 193601 (2018).

[35] M. O. Scully, E. S. Fry, C. H. Ooi, and K. Wódkiewicz, Directed spontaneous emission from an extended ensem- 
ble of $\mathrm{N}$ atoms: Timing is everything, Phys. Rev. Lett. 96, 10501 (2006).

[36] R. Khomeriki and S. Flach, Landau-Zener Bloch oscillations with perturbed flat bands, Phys. Rev. Lett. 116, 245301 (2016).

[37] See Supplemental Material, which includes Refs. 25, 3840.

[38] D. W. Wang, H. T. Zhou, M. J. Guo, J. X. Zhang, J. Evers, and S. Y. Zhu, Optical diode made from a moving photonic crystal Phys. Rev. Lett. 110, 093901 (2013).

[39] F. A. An, E. J. Meier, J. Ang'ong'a, and B. Gadway, Correlated dynamics in a synthetic lattice of momentum states, Phys. Rev. Lett. 120, 040407 (2018).

[40] D. Xie, T. S. Deng, T. Xiao, W. Gou, T. Chen, W. Yi, and B. Yan, Topological quantum walks in momentum space with a Bose-Einstein condensate, Phys. Rev. Lett. 124, 050502 (2020).

[41] T. Jacqmin, I. Carusotto, I. Sagnes, M. Abbarchi, D. D. Solnyshkov, G. Malpuech, E. Galopin, A. Lemaître, J. Bloch, and A. Amo, Direct Observation of Dirac cones and a flatband in a honeycomb lattice for polaritons Phys. Rev. Lett. 112, 116402 (2014).

[42] R. Drost, T. Ojanen, A. Harju, and P. Liljeroth, Topological states in engineered atomic lattices, Nat. Phys. 13, 668 (2017).

[43] M. R. Slot, T. S. Gardenier, P. H. Jacobse, G. C. van Miert, S. N. Kempkes, S. J. Zevenhuizen, C. M. Smith, D. Vanmaekelbergh, and I. Swart, Experimental realization and characterization of an electronic Lieb lattice, Nat. Phys. 13, 672 (2017).

[44] H. Cai, J. Liu, J. Wu, Y. He, S. Y. Zhu, J. X. Zhang, and D. W. Wang, Experimental observation of momentumspace chiral edge currents in room-temperature atoms, Phys. Rev. Lett. 122, 023601 (2019).

[45] M. Atala, M. Aidelsburger, M. Lohse, J. T. Barreiro, B. Paredes, and I. Bloch, Observation of chiral currents with ultracold atoms in bosonic ladders, Nat. Phys. 10, 588 (2014).

[46] M. Mancini, G. Pagano, G. Cappellini, L. Livi, M. Rider, J. Catani, C. Sias, P. Zoller, M. Inguscio, M. Dalmonte, L. Fallani, Observation of chiral edge states with neutral fermions in synthetic Hall ribbons, Science 349, 1510 (2015).

[47] B. Stuhl, H. I. Lu, L. Aycock, D. Genkina, and I. Spiel- man, Visualizing edge states with an atomic Bose gas in the quantum Hall regime, Science 349, 1514 (2015).

[48] L. F. Livi, G. Cappellini, M. Diem, L. Franchi, C. Clivati, M. Frittelli, F. Levi, D. Calonico, J. Catani, M. Inguscio, and L. Fallani, Synthetic dimensions and spin-orbit coupling with an optical clock transition, Phys. Rev. Lett. 117, 220401 (2016).

[49] E. Anisimovas, M. Račiūnas, C. Sträter, A. Eckardt, I. B.Spielman, and G. Juzeliūnas, Semisynthetic zigzag optical lattice for ultracold bosons, Phys. Rev. A 94, 063632 (2016).

[50] F. A. An, E. J. Meier, and B. Gadway, Direct observation of chiral currents and magnetic reflection in atomic fluxLattices, Sci. Adv. 3, e1602685 (2017).

[51] A. Dutt, Q. Lin, L. Yuan, M. Minkov, M. Xiao, and S. Fan, A single photonic cavity with two independent physical synthetic dimensions, Science 367, 59 (2020).

[52] T. Ozawa and I. Carusotto, Anomalous and quantum Hall effects in lossy photonic lattices, Phys. Rev. Lett. 112, 133902 (2014).

[53] D. W. Wang, S. Y. Zhu, J. Evers, and M. O. Scully, Highfrequency light reflector via low-frequency light control, Phys. Rev. A 91, 011801 (2015).

[54] N. Masumoto, N. Y. Kim, T. Byrnes, K. Kusudo, A. Löffler, S. Höfling, A. Forchel, and Y. Yamamoto, Exciton-polariton condensates with flat bands in a twodimensional kagome lattice, New J. Phys. 14, 065002 (2012).

[55] S. Klembt, T. H. Harder, O. A. Egorov, K. Winkler, H. Suchomel, J. Beierlein, M. Emmerling, C. Schneider, and S. Höfling, Polariton condensation in S- and P-flatbands in a two-dimensional Lieb lattice, Appl. Rev. Lett. 111, 231102 (2017).

[56] C. Whittaker, E. Cancellieri, P. Walker, D. Gulevich, H. Schomerus, D. Vaitiekus, B. Royall, D. Whittaker, E. Clarke, I. Iorsh, I. A. Shelykh, M. S. Skolnick, and D. N. Krizhanovskii, Exciton polaritons in a twodimensional lieb lattice with spin-orbit coupling, Appl. Phys. Lett. 120, 097401 (2018).

[57] Y. Kuno, T. Orito, and I. Ichinose, Flat-band many-body localization and ergodicity breaking in the Creutz ladder, New J. Phys. 22, 013032 (2020).

[58] H. Tasaki, Ferromagnetism in the Hubbard models with degenerate single-electron ground states, Phys. Rev. Lett. 69, 1608 (1992). 